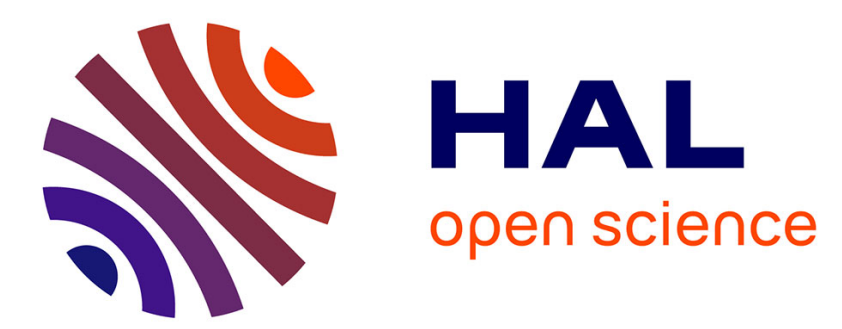

\title{
RECENT DEVELOPMENTS IN THE SPECTROSCOPY OF RARE EARTH MONOXIDES
}

\author{
C. Linton
}

\section{To cite this version:}

C. Linton. RECENT DEVELOPMENTS IN THE SPECTROSCOPY OF RARE EARTH MONOXIDES. Journal de Physique IV Proceedings, 1991, 01 (C7), pp.C7-525-C7-528. 10.1051/jp4:19917138 . jpa-00250811

\section{HAL Id: jpa-00250811 https://hal.science/jpa-00250811}

Submitted on 1 Jan 1991

HAL is a multi-disciplinary open access archive for the deposit and dissemination of scientific research documents, whether they are published or not. The documents may come from teaching and research institutions in France or abroad, or from public or private research centers.
L'archive ouverte pluridisciplinaire HAL, est destinée au dépôt et à la diffusion de documents scientifiques de niveau recherche, publiés ou non, émanant des établissements d'enseignement et de recherche français ou étrangers, des laboratoires publics ou privés. 


\title{
RECENT DEVELOPMENTS IN THE SPECTROSCOPY OF RARE EARTH MONOXIDES
}

\author{
C. LINTON \\ Physics Department, University of New Brunswick, Fredericton, N.B. E3B 5A3, Canada
}

\begin{abstract}
Recent work on the Spectroscopy and structure of rare earth oxide molecules is reviewed. Various techniques used to examine specific aspects of the electronic structure and test the ligand field theoretical eigenvectors are discussed and illustrated with results obtained for specific molecules.
\end{abstract}

1. Introduction

At the first M2P conference, I reviewed the work done on the Spectroscopy of diatomic rare earth oxide (REO) molecules up to that time (1). The review concentrated on outlining the ligand field theory (LFT) method used to explain the experimental results in terms of electron configuration and discussed the assignments of electronic states and the linkages between them for several molecules. The use of hyperfine structure as a guide to the configuration was also briefly discussed. In the past few years, the study of these molecules has continued and expanded. Although the crucial determination of energy linkages using low or medium resolution dispersed fluorescence has continued, it was felt that the low lying state superconfigurations had been well established and more emphasis was placed on high resolution studies aimed at testing the theory in more detail. These include examination of rotational structure, $\Omega$-doubling, hyperfine structure, Zeeman effect and Stark effect. Each of these serves as a diagnostic tool enabling us to study a particular aspect of the theory and allows us to test both global predictions, such as energy level diagrams, and finer details such as the ligand field eigenvectors. This article will outline the recent REO results by reviewing briefly the basic ideas of LFT as applied to REO's and discussing the various diagnostic tools mentioned above, and the way in which they probe the molecular structure. This article is not intended as a compendium of molecular constants, energy levels or a detailed study of individual molecules, all of which can be found in the literature. Detailed results on individual molecules are used as examples to illustrate the points being discussed. All experiments involved laser spectroscopy with single mode dye lasers. Experimental details are given in the references.

Ligand Field Theory: This is applied to gas phase REO's in an attempt to explain, in a relatively simple manner, the structure and energy patterns arising from low lying superconfigurations of molecules containing unfilled f-shells which lead to an extraordinarily large number of close lying electronic states and horrendous complexity in the spectrum. The basic idea is that the molecules are ionically bonded, $\mathrm{M}^{+2} \mathrm{O}^{-2}$, and the molecular states arise as a result of the atomic ion states being perturbed by the field of the $\mathrm{O}^{-2}$ ligand. Thus, the metal ion quantum numbers remain good in the 
molecule and the only truly molecular quantum number is $\Omega$, the projection of the electron angular momentum on the internuclear axis. Theoretical calculations $(2,3)$ have shown that the ground state superconfigurations are expected to be $\mathrm{M}^{+2}\left(4 \mathrm{f}^{\mathrm{N}-1} 6 \mathrm{~s}\right) \mathrm{O}^{-2}$ except for EuO and $\mathrm{YbO}$ in which the metal ion configurations are $\mathrm{f}^{7}$ and $\mathrm{f}^{14}$ respectively. The quantum numbers describing the molecular states are $J_{c}$, the total angular momentum of the $f$-electron "core", $J_{a}$ the total angular momentum of the metal ion $\left(J_{i}=J_{c} \pm 1 / 2\right)$, and $\Omega$. The eigenvectors of the electronic states are written as linear combinations of the basis functions $J_{c}, J_{a}, \Omega>$ and have been listed by Carette and Hocquet (3) for all REO's.

\section{Diagnostic Tools}

Vibrational Frequencies: These can be obtained from low resolution spectra and are a quick and easy guide to distinguishing between electron configurations, particularly $\mathrm{f}^{\mathrm{N}-1} \mathrm{~s}$ and $\mathrm{f}^{\mathrm{N}}$. This diagnostic property arises from the fact that the 4 f orbitals are compact and shield the nuclear charge from the oxygen whereas the $6 \mathrm{~s}$ orbital is large and allows the oxygen to see the nuclear charge. Thus, for $\mathrm{M}^{+2}\left(\mathrm{f}^{\mathrm{N}-1} \mathrm{~s}\right)$, the oxygen will see a charge of +3 whereas, for $\mathrm{f}^{\mathrm{N}}$, all the electrons are shielding and the oxygen sees a charge of +2 . The force constant and hence the vibrational frequency will therefore be greater for $\mathrm{f}^{\mathrm{N}-1} \mathrm{~s}$ than $\mathrm{f}^{\mathrm{N}}$. Our results have shown that all the REO low lying states have vibrational frequencies of around $820-830 \mathrm{~cm}^{-1}$ except for the ground states of $\mathrm{EuO}$ and $\mathrm{YbO}$ whose frequencies are $-690 \mathrm{~cm}^{-1}(4,5)$. In $\mathrm{YbO}$, we observed an $\mathrm{f}^{14}$ ground state $\left(\omega-690 \mathrm{~cm}^{-1}\right)$ and a group of low lying states with $\omega-830 \mathrm{~cm}^{-1}$ which must belong to $\mathrm{f}^{13} \mathrm{~s}$. This provides the only linkage between $\mathrm{f}^{\mathrm{N}}$ and $\mathrm{f}^{\mathrm{N}-\mathrm{S}} \mathrm{s}$ configurations so far observed (5). This work was particularly important in resolving a discrepancy in predictions made by LFT theory and a pseudo potential approach (7).

Energy Linkages: The $\Omega$ assignments and energy patterns are important in establishing the superconfiguration. The ground state $\Omega$ is easily determined from the configuration and the energy patterns have been predicted theoretically $(2,3)$. For example, $\mathrm{SmO}$ has an $\mathrm{f}^{5} \mathrm{~s}$ configuration. The $\mathrm{f}^{5}$ core gives a ${ }^{6} \mathrm{H}_{2.5}$ lowest state with $\mathrm{J}_{\mathrm{c}}=2.5$. Interaction with the $\mathrm{s}$ electron gives $\mathrm{J}_{\mathrm{a}}=2$ and 3 with $\mathrm{J}_{\mathrm{a}}=2$ lying lower. Interaction with the ligand field of $\mathrm{O}^{-2}$ gives a ground state with $\mathbf{\Omega}=0^{-}$, precisely as observed. Twelve low lying SmO states have been assigned and the energy pattern is in very good agreement with LFT predictions (8).

Rotational Structure: High resolution laser excitation spectroscopy enabled us to obtain rotationally resolved spectra of all REO's and derive molecular constants, test isotope dependence and, in particular, observe the first lines to establish definitively the $\Omega$ assignment for the states. Many perturbations were observed which sometimes facilitated assigning the lines and, in the case of SmO (8), enabled us to completely assign two very close interacting states.

2-Doubling: The $\Omega$ doubling in REO's, especially in $\Omega=1$ states, is very large and, for the heavier molecules, ErO and TmO, is larger than the rotational spacing. The large doubling is a result of the fact that, in many cases, the low $\Omega$ molecular states are projections on the internuclear axis of free-ion atomic states of high angular momentum, $\mathbf{J}_{\mathbf{2}}$.

For $\Omega=1$ states, the doubling is caused by interaction with neighbouring $\Omega=0$ states and is determined by the matrix element of the $\mathrm{J}_{\mathrm{a}}$ term in the Hamiltonian. This gives the usual $q \mathrm{~J}(\mathrm{~J}+1)$ term for the level splitting where $q$ is given by

$$
q=2 B^{2} J_{a}\left(J_{a}+1\right) / \Delta E_{10}
$$

where $\Delta E_{10}$ is the separation of the interacting $\Omega=1$ and 0 states. For REO's, where $J_{a}$ is often large, this leads to larger than normal $\Omega$-doubling. This usually manifests itself as an easily detectable difference between $R Q$ and $Q P$ branch separations in transitions from the same $J$ level as seen in 
many observed transitions in $\mathrm{CeO}(9)$ and $\mathrm{SmO}(8)$.

The $\Omega$ doubling is a sensitive test of the eigenvectors of interacting $\Omega=0$ and 1 states. The eigenvectors are linear combinations of basis functions of different $\mathrm{J}_{\mathrm{c}}$ and $\mathrm{J}_{\mathrm{a}}$ and thus the $\Omega$-doubling constant will be the sum of terms of different $J_{a}$ whose value will depend on the coefficients of the basis functions. As these have all been listed (3), $\Omega$ doubling constants can be calculated and compared with experiment. This has been done extensively for SmO (8) where it was shown that the eigenvectors reproduced experimental $\Omega$-doubling very well for the lowest two $\Omega=1$ states but the agreement was much worse for higher $\Omega=1$ states (8). This clearly indicates the need for refinement of the theory.

In ErO, the ground state is predicted to be $\Omega=0^{-}$with $\mathrm{J}_{\mathrm{a}}=8$. The energy separation of the lowest $\Omega=0$ and 1 states is predicted to be $-25 \mathrm{~cm}^{-1}$. This will lead to extremely large $\Omega$-doubling in which the doubling will be greater than the rotational spacing and the $\mathrm{Q}$ branch, instead of lying between the $R$ and $P$ branches at given $J$, will be at higher or lower frequency than both branches and will get further from these branches as $\mathrm{J}$ increases, giving the band a very unusual appearance. This has been clearly observed in preliminary ErO experiments although the results have not yet been analyzed.

In $\mathrm{TmO}$, the predicted ground state has $\Omega=0.5$ and $\mathrm{J}_{\mathrm{a}}=6.5$. As well as the $\Omega$-doubling caused by interaction of 0.5 and 1.5 states, there is also a splitting of the levels in a 0.5 state caused by a parity dependent matrix element $\left\langle\Omega=0.5 \mathrm{~J}_{\mathrm{a}}^{+} \mathrm{J} \Omega=-0.5>\right.$ which is off-diagonal in the signed basis functions but diagonal in the symmetrized e,f parity basis sets. Because of the large $J_{a}$, this will cause a very large ef splitting, greater than the rotational spacing, and result in an isolated $Q$ branch as described above. This has been recently observed in resolved fluorescence and clearly indicates the presence of a low lying $\Omega=0.5$ state.

Hyperfine Structure: This is a sensitive probe of the orbital structure of electronic states. For example, the ground configuration of most REO's is $\mathrm{f}^{\mathrm{N}-1} \sigma$. The hyperfine splitting will be a combination of an orbital contribution for the f-electrons and a Fermi-contact term from the $s$ electron. For both $\mathrm{PrO}(10)$ and $\mathrm{HoO}(11)$, the felectron contribution was found to be the same as in the free-ion while the $s$ electron contribution was reduced by $-50 \%$. This indicates that the compact f-orbitals are unaffected by molecule formation and that the $\sigma$ orbital is approximately a 50-50 mixture of $6 \mathrm{~s}$ and $6 \mathrm{p}$ orbitals. We have recently observed resolved hyperfine structure in HoO up to $\mathrm{J}=60$ and in ${ }^{161} \mathrm{DyO}$ and ${ }^{163} \mathrm{DyO}$. Analysis is in progress.

Zeeman Effect: The Zeeman effect serves as a check on the $J$ and $\Omega$ assignments and enables us to determine Landé $g$ factors for the electronic states. These can also be calculated from the eigenvectors and serve as a test of the calculations. For $\mathrm{CeO}$ (12), calculated and observed g values were in good agreement whereas for SmO (8), the agreement was good only for the lowest states. Both this and the $\Omega$-doubling point to a need for more refined calculations of higher states of the ground superconfiguration.

Stark Effect: We have recently observed the Stark effect in DyO by applying electric fields up to $14 \mathrm{kV} / \mathrm{cm}$. Because the transition was $\Omega=7-8$, the lowest J's are 7 and 8 giving 15 and 17 components respectively in an electric field. The spectra are therefore complex, but they have been successfully assigned and shown that the dipole moment of the ground state is 4.6 Debye. This shows that there is a strong polarization of the metal ion $6 s 6 p$ hybrid orbital away from the oxygen (13). Further dipole moment measurements should prove very useful in determining the orbital structure and polarization in REO's. 


\section{Conclusions}

The above summary has shown how different Spectroscopic tools have been used to examine various aspects of the electronic structure of REO's and to test the extent to which present LFT calculations can explain the observations. The results highlight areas in which further calculations are needed. Present experiments include detailed investigation of hyperfine structure of $\mathrm{HoO}$ and DyO, determination of energy linkages and structure of $\mathrm{ErO}$ and TmO, and Stark effect measurements of SmO. An investigation of the rare earth halides has just started and the structure of HoF is being analyzed.

\section{References}

1. C. Linton, J. de Physique, 48, C7:611-615 (1987)

2. M. Dulick, E. Murad, and R.F. Barrow, J. Chem. Phys. 85, 385-390 (1986)

3. P. Carette and A. Hocquet, J. Mol. Spectrosc. 131, 301-324 (1988)

4. S.A. McDonald, Ph.D. Thesis, MIT, 1985

5. C. Linton, S. McDonald, S. Rice, M. Dulick, Y.C. Liu and R.W. Field, J. Mol. Spectrosc. 101, 332-343 (1983)

6. S.A. McDonald, S.F. Rice, R.W. Field and C. Linton, J. Chem. Phys. 93, 7676-7868 (1990)

7. M. Dolg and H. Stoll, Theor. Chim. Acta 75, 369-387 (1989)

8. Guo Bujin and C. Linton, J. Mol. Spectrosc. 147, 120-141 (1991)

9. C. Linton, M. Dulick, R.W. Field, P. Carette, P.C. Leyland and R.F. Barrow, J. Mol. Spectrosc. 102, 441-497 (1983)

10. M. Dulick and R.W. Field, J. Mol. Spectrosc., 113, 105-141 (1985)

11. C. Linton and Y.C. Liu, J. Mol. Spectrosc., 131, 367-381 (1988)

12. H. Schall, J.A. Gray, M. Dulick and R.W. Field, J. Chem. Phys. 85, 751-762 (1986)

13. C. Linton and B. Simard, Submitted to J. Chem. Phys. 